\title{
Thalamic dopamine D2-receptor availability in schizophrenia: a study on antipsychotic-naive patients with first-episode psychosis and a meta-analysis
}

\author{
Pontus Plavén-Sigray ${ }^{1,2,10}$, Pauliina Ikonen Victorsson ${ }^{1,10}$, Alexander Santillo ${ }^{1,3}$, Granville J. Matheson ${ }^{1,4,5}$, Maria Lee (iD ${ }^{1}$, Karin Collste ${ }^{1}$, \\ Helena Fatouros-Bergman ${ }^{1}$, Carl M. Sellgren ${ }^{1,6}$, Sophie Erhardt $\mathbb{D}^{6}{ }^{6}$, Ingrid Agartz ${ }^{1,7,8}$, Christer Halldin ${ }^{1}$, Lars Farde $\mathbb{D}^{1}$ and $^{1}$ \\ Simon Cervenka (iD) ${ }^{1,9 凶}$
}

(c) The Author(s) 2021

Pharmacological and genetic evidence support a role for an involvement of the dopamine D2-receptor (D2-R) in the pathophysiology of schizophrenia. Previous molecular imaging studies have suggested lower levels of D2-R in thalamus, but results are inconclusive. The objective of the present study was to use improved methodology to compare D2-R density in whole thalamus and thalamic subregions between first-episode psychosis patients and healthy controls. Differences in thalamocortical connectivity was explored based on the D2-R results. 19 antipsychotic-naive first-episode psychosis patients and 19 age- and sex-matched healthy controls were examined using high-resolution Positron Emission Tomography (PET) and the high-affinity D2-R radioligand $\left[{ }^{11} \mathrm{C}\right]$ FLB457. The main outcome was D2-R binding potential $\left(\mathrm{BP}_{\mathrm{ND}}\right)$ in thalamus, and it was predicted that patients would have lower binding. Diffusion tensor imaging (DTI) was performed in a subgroup of 11 patients and 15 controls. D2-R binding in whole thalamus was lower in patients compared with controls (Cohen's dz $=-0.479, p=0.026$, Bayes Factor (BF) $>4$ ). Among subregions, lower $\mathrm{BP}_{\mathrm{ND}}$ was observed in the ROI representing thalamic connectivity to the frontal cortex (Cohen's $\mathrm{dz}=-0.527, p=0.017, \mathrm{BF}>$ 6). A meta-analysis, including the sample of this study, confirmed significantly lower thalamic D2-R availability in patients. Exploratory analyses suggested that patients had lower fractional anisotropy values compared with controls (Cohen's $d=-0.692$, $p=0.036)$ in the inferior thalamic radiation. The findings support the hypothesis of a dysregulation of thalamic dopaminergic neurotransmission in schizophrenia, and it is hypothesized that this could underlie a disturbance of thalamocortical connectivity.

Molecular Psychiatry (2022) 27:1233-1240; https://doi.org/10.1038/s41380-021-01349-x

\section{INTRODUCTION}

The dopamine system has for several decades been of central importance for research on the pathophysiology and treatment of schizophrenia. Well over one hundred molecular imaging studies have investigated the in vivo effects of antipsychotic treatments, showing that all currently licensed antipsychotic drugs block dopamine D2 receptors (D2-R) [1, 2]. Moreover, a causal role for $D 2-R$ in the disease mechanism of schizophrenia has been suggested by genetic studies [3]. Molecular imaging studies have shown a slight increase in striatal D2-R in psychosis and schizophrenia patients compared with controls, although this was not significant in the subsample of antipsychotic-naive firstepisode patients [4]. However several other brain regions have been considered more important in generating the diversity of symptoms observed in schizophrenia. Further investigations of dopaminergic function outside of the striatum is therefore needed in order to understand the pathophysiology of the disorder.
A region of key interest in schizophrenia is the thalamus, which is richly interconnected to the cortex, and plays a central role in coordinating signaling both to and in between cortical regions $[5,6]$. In a series of positron emission tomography (PET) studies using the high-affinity D2-R radioligands $\left[{ }^{11} \mathrm{C}\right] \mathrm{FLB} 457$ and $\left[{ }^{18} \mathrm{~F}\right]$ Fallypride, which were developed for low-density extrastriatal regions $[7,8], D 2-R$ binding has shown to be lower in thalamus in patients with psychosis compared with controls [9-12]. However, no differences, or increases have also been observed using these radioligands [13-16]. A meta-analysis, which also included studies using other radioligands, showed lower thalamic D2-R binding in patients $(d=-0.32)$, but the difference was not statistically significant [17]. Importantly, the analysis included several studies with patients with previous exposure to medication and in later disease stages, and some of the studies used radioligands with low signal-to-noise for quantifying $D 2-R$ in extrastriatal brain regions. Hence, additional studies are needed to confirm if there

\footnotetext{
${ }^{1}$ Centre for Psychiatry Research, Department of Clinical Neuroscience, Karolinska Institutet and Stockholm Health Care Services, Region Stockholm, Stockholm, Sweden. ${ }^{2}$ Neurobiology Research Unit, Copenhagen University Hospital, Rigshospitalet, Copenhagen, Denmark. ${ }^{3}$ Clinical Memory Research Unit, Department of Clinical Sciences, Lund University, Malmö, Sweden. ${ }^{4}$ Molecular Imaging and Neuropathology Division, Columbia University, New York, NY, USA. ${ }^{5}$ Department of Biostatistics, Columbia University, New York, NY, USA. ${ }^{6}$ Department of Physiology and Pharmacology, Karolinska Institutet, Stockholm, Sweden. ${ }^{7}$ Norwegian Centre for Mental Disorders Research (NORMENT), Institute of Clinical Medicine, University of Oslo, Oslo, Norway. ${ }^{8}$ Department of Psychiatric Research, Diakonhjemmet Hospital, Oslo, Norway. ${ }^{9}$ Department of Neuroscience, Psychiatry, Uppsala University, Uppsala, Sweden. ${ }^{10}$ These authors contributed equally: Pontus Plavén-Sigray, Pauliina Ikonen Victorsson. ${ }^{凶}$ email: simon.cervenka@neuro.uu.se
} 
indeed is a decrease of D2-R in thalamus in schizophrenia, preferably using high-affinity radioligands in samples not affected by antipsychotic drug treatment and other confounders stemming from chronical illness.

The thalamic complex is functionally heterogenous, being part of parallel circuits with distinct physiological implications. Neuropathological studies in schizophrenia have primarily shown a significant reduction in the number of neurons in the thalamic mediodorsal and pulvinar subregions [18-20]. In some of the previous PET studies, low D2-R binding was found specifically in medial [9-12] and posterior subregions of the thalamus [10]. However, the low resolution of the PET systems used in these studies and the diversity in region of interest (ROI) definition limit the conclusions that can be drawn. Additionally, none of the previous studies based the regional analysis on underlying functional anatomy.

The primary aims of this study were to (1) confirm previous observations of lower D2-R binding in the thalamus in psychosis and schizophrenia patients, (2) extend the subregional D2-R analysis using improved methodology, and (3) based on subregional D2-R differences, investigate aberrations in thalamo-cortical connectivity in an exploratory manner. For these purposes, we used highresolution PET and the high-affinity radioligand $\left[{ }^{11} \mathrm{C}\right] \mathrm{FLB} 457$ to examine 19 drug-naive first-episode psychosis patients and 19 matched healthy control subjects. Thalamic subregions were definied using a connectivity-based atlas. An exploratory diffusion tensor imaging (DTI) analysis of thalamic structural connectivity was performed based on the PET results for a subgroup of individuals.

\section{SUBJECTS AND METHODS \\ Patients and healthy control subjects}

The study was approved by the Stockholm Regional Ethics Committee (Dnr 2010/879-31/1) and the Radiation and Safety Committee at the Karolinska University Hospital. All subjects provided written consent after receiving a complete description of the study, according to the Helsinki declaration. Patients with first-episode psychosis, as defined by first contact with psychiatric services due to psychotic symptoms, were recruited from outpatient clinics and psychiatric wards in the Stockholm region. At time of recruitment and during their participation in the study, all patients were fully naive to antipsychotic drugs. The final patient sample consisted of 19 subjects ( 11 males, 8 females, mean (SD) age 29.3 (6.3)). Patients fulfilled criteria for the following Diagnostic and Statistical Manual of Mental Disorders IV diagnoses at inclusion: schizophrenia $(N=6)$, schizophreniform disorder $(N=5)$, psychotic disorder NOS $(N=5)$, and delusional disorder $(N=3)$, as assessed using SCID-I. Symptoms were rated using the Positive And Negative Syndrome Scale (PANSS).

Nineteen healthy control subjects ( 11 males, 8 females, mean (SD) age 29.2 (5.9)) were recruited by advertisement to match against a corresponding patient of the same age $( \pm 2$ years $)$ and the same sex. Control subjects were healthy according to medical history, clinical examination, routine laboratory blood test as well as a brain magnetic resonance imaging (MRI) examination.

Two patients and two healthy control subjects used nicotine on a daily basis. Eight patients received anxiolytics (benzodiazepine $(5$ patients, oxazepam, mean $=11 \mathrm{mg}$, range $5-15 \mathrm{mg}$ ) and/or antihistamines (5 patients)) during their participation in the PET study. For exclusion criteria see Supplementary information.

\section{MRI, DTI examinations, and ROI delineation}

Structural MRI data was obtained using a 3-T General Electric Discovery MR750 system (GE, Milwaukee, WI, USA). T1-weighted images were used for ROI delineation, and T2-weighted FLAIR images were assessed for pathology by a neuroradiologist. One control subject was examined on a 1.5T GE Signa system (Milwaukee, WI) instead of the 3 T-system.

Diffusion-weighted data were collected for a subset of 11 patients and 15 control subjects with a spin-echo sequence in the axial plane with diffusion gradients in 60 directions and $b$-values of $1000 \mathrm{~s} / \mathrm{mm}^{2}$. Images were acquired with a field of view of $240 \mathrm{~mm}$ and a slice thickness of $290 \mathrm{~mm}$, with a voxel size of $1.875 \times 1.875 \times 2.9 \mathrm{~mm}$.

Thalamic ROIs were obtained using the FMRIB FSL v.5.0.7 software [21]. For whole thalamus ROI, the FSL Harvard-Oxford subcortical atlas was used with a $50 \%$ probability threshold. For thalamic subregionals delineation, the Oxford Thalamic Connectivity Atlas [22] (probability thresholding at $50 \%$ ) was used to extract the following ROls: (1) Anterior part of the mediodorsal nucleus and ventral anterior nuclei with DTI projections to the prefrontal cortex (THA-PFC ROI); (2) the most medial part of the mediodorsal nucleus, parts of the anterior complex and medial and inferior pulvinar that projects to the temporal cortex (THA-TC); (3) ventral posterolateral nucleus which projects to primary motor cortex (THA-M1); (4) ventral lateral nucleus and the ventral anterior nucleus which project to premotor areas (THA-PreMC); and (5) anterior parts of the pulvinar that project to posterior parietal cortex (THA-PPC) (Fig. 1). For additional details see Supplementary information. Since we had no hypothesis of hemispheric differences, right and left hemisphere ROls were pooled to increase count statistics.

A cerebellum mask was also extracted using the maximum probability FSL MNI FNIRT atlas. The mask was then trimmed to exclude voxels close to the vermis, the cortex, and the most inferior part of the PET image, as has been described previously [23]. Subsequently, all ROls were warped back into the individual T1-image space.

\section{PET examinations and data quantification}

All patients and all healthy controls were examined using the highresolution research tomograph (Siemens Molecular Imaging, Knoxville, TN, USA) at the Karolinska Institutet PET center. A drug toxicology screen was performed prior to the PET examination. $\left[{ }^{11} \mathrm{C}\right] \mathrm{FLB} 457$ was prepared as described previously [24] and injected into the antecubital vein as a rapid bolus. Mean (SD) injected activity was 425.95 (47.98) MBq, mean (SD) specific activity was 374 (203) Gbq/micromol, and mean injected mass was 0.4-0.7 microg. All emission scans were 90 min long. Non-invasive Logan Graphical Analysis [25] with cerebellum as reference region was used to obtain $\mathrm{BP}_{\mathrm{ND}}$ values in all thalamic ROIs. All quantitative analyses were performed using the "kinfitr" package in R [26]. For additional details, see Supplementary information.

\section{Exploratory DTI analysis}

The thalamus and its subregions (i.e., as defined by Oxford Thalamic Connectivity Atlas, see above), connect to cortical regions by axons gathered into white matter tracts, radiations. Based on the results of the PET analyses of thalamic subregions we chose to study the two major radiations leading to and from the TH-PFC ROI, the anterior thalamic radiation (ATR) and inferior thalamic radiation (ITR) $[22,27]$. This was done by manual dissection according to existing protocols $[28,29]$ on whole brain tractography with a fractional anisotropy threshold of 0.2 and an angle threshold of $30^{\circ}$, using ExploreDTI [30]. See Supplementary information and Supplementary Fig. 1 for a detailed description and illustration. Motion and eddy current correction of the data was performed using ElastiX [31]. Diffusion-weighted sequences were only available for a subset of the study subjects (11 patients and 15 healthy controls).

\section{Statistical analysis}

In this study, the statistical analyses and procedures related to analysis of PET data (whole thalamus and subthalamic analyses) were pre-registered a priori and are hence considered to be confirmatory analyses. The DTI analyses were considered to be exploratory in nature, with the aim of generating new hypotheses regarding connectivity changes associated with an aberrant thalamic D2-R system. Since previously reported effect sizes of patient-control differences in thalamic D2-R availability have been small to medium in size [17], we elected to form directed (i.e. one-sided) a priori hypotheses in this study, in conjunction with paired matching of patients and controls, in order to maximize the power of the tests. This entails us making more specific predictions about the effect, which affords us a higher degree of sensitivity, assuming that these predictions were appropriate. In this way, we are leveraging the findings of prior research to improve the power of our research design while minimizing the number of participants who must be exposed to radioactivity to test these hypotheses. The preregistration protocol (https://osf.io/nhr3w/) further outlines the a priori predictions and the rationale behind the chosen statistical tests.

To examine patient-control differences in $\left[{ }^{11} \mathrm{C}\right] \mathrm{FLB} 457 \mathrm{BP}_{\mathrm{ND}}$, we performed a frequentist paired-sample $t$-test using a one-sided hypothesis, i.e. expecting patients to have lower $\left[{ }^{11} \mathrm{C}\right] \mathrm{FLB} 457 \mathrm{BP}_{\mathrm{ND}}$ (specified a priori in the preregistration document). We examined the whole thalamus as well as thalamic subregions (setting $a=0.05$ ). We also applied a Bayesian 


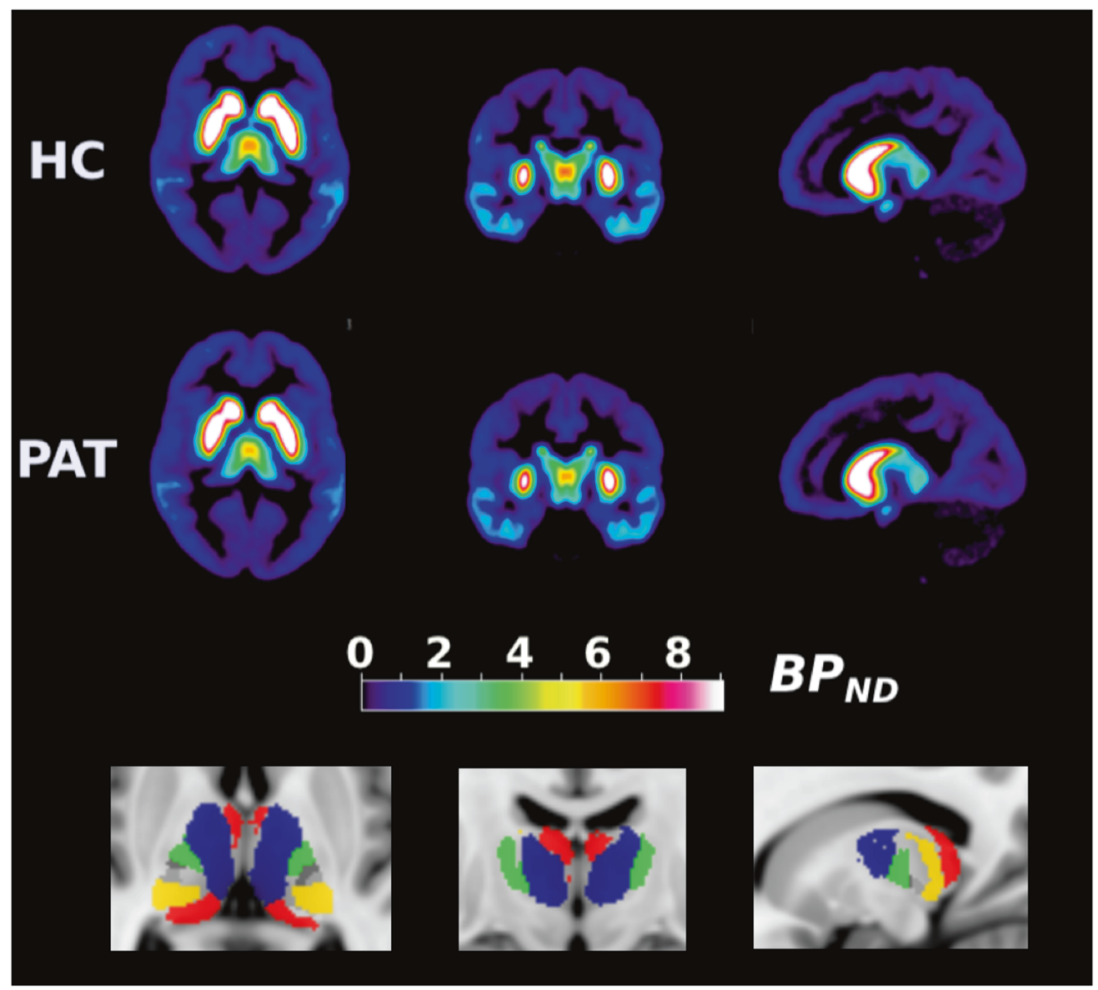

Fig. 1 Average $\mathbf{B P}_{\mathrm{ND}}$ values and thalamic ROls. Upper panel: average $\mathrm{BP}_{\mathrm{ND}}$ image values for first-episode psychosis patients and matched control subjects. Lower panel: ROls for thalamic subregions shown on a template T1 MR image, based on their connectivity to cortical brain regions. Blue $=$ THA-PFC (projections to prefrontal cortex), red $=$ THA-TC (temporal cortex), gray $=$ THA-M1 (primary motor cortex), green $=$ THA-PreMC (premotor cortex), orange = THA-PPC (posterior parietal cortex).

paired-sample $t$-test, in which we made use not only of the expected direction of the effect, but also the expected magnitude observed in previous studies. This was done using a meta-analytical effect size on D2-R patient-control differences in thalamus and its uncertainty as a prior over the research hypothesis, which we compared to the null hypothesis of no difference [32] (see Supplementary information for details). This approach, also specified a priori in the preregistration document, allows us to make even more specific predictions compared to the frequentist approach, by taking previously available information into account when shaping the research hypothesis. By using the Bayes Factor (BF) we then evaluated whether the collected data are more consistent with the hypothesis of decreases in thalamic D2-R binding in psychosis and schizophrenia patients $\left(\mathrm{H}_{1}\right)$, or with the null hypothesis of no effect $\left(\mathrm{H}_{0}\right)$. A BF in favor of $\mathrm{H} 1$ relative to $\mathrm{HO}\left(\mathrm{BF}_{\mathrm{H} 1 \mathrm{HO}}\right)$ above 3 is commonly interpreted as moderate evidence in favor of the alternative hypothesis, and in the present study would be viewed as support for a successful replication of the previous findings of lower patient binding. $\mathrm{A} \mathrm{BF}$ in favor of $\mathrm{H} 0$ relative to $\mathrm{H} 1\left(\mathrm{BF}_{\mathrm{HO}: \mathrm{H} 1}\right)$ above 3 is commonly interpreted as moderate evidence in favor of the null hypothesis, indicating that no difference in binding between patients and controls gained support in our study [33].

To correct for multiple comparisons in the subregional analysis, a permutation procedure was employed [34] to estimate the average increase in type I error, while taking the dependency between the five ROls into account, resulting in a family-wise error corrected $a$ of 0.0356 (see Supplementary information for additional details).

The statistical analyses presented in the main text deviates from the preregistration in one important manner. When defining the alternative hypothesis for the BF test of whole-thalamus difference above, we opted to also include the study by Talvik et al. [12], Yasuno et al., Slifstein et al. [13], and Veselinovic et al. [15] in addition to the studies reported in the Kambeitz et al. meta-analysis [17]. A systematic literature search was performed dating from January 1st 2013 to March 16th 2021 to make sure no additional studies were overlooked, allowing us to shape an $\mathrm{H}_{1}$ informed by all previous published literature (see Supplementary information and Supplementary Fig. 2 for additional details). For full transparency the results from the BF-analysis carried out as written in the preregistration are also reported in the Supplementary information (the overall conclusion does not differ between these two analyses). In the results section, we consequently opted to present meta-analytic results on thalamic D2-R availability differences between patients with psychotic disorders and healthy controls, combining the results from this study with the previous literature (based on the literature search above). One analysis was conducted using all published studies, similar to Kambeitz et al., and we also performed a separate analysis including only studies using either of the two high-affinity radioligands $\left[{ }^{18} \mathrm{~F}\right]$ Fallypride or $\left[{ }^{11} \mathrm{C}\right] \mathrm{FLB} 457$, which have higher sensitivity for low-density D2-R regions than all other radioligands used. A further addition to the preregistration protocol is that frequentist and BF two-sample $t$-tests were also performed (presented in the supplementary tables), in addition to the paired $t$-tests. All statistical analyses were carried out using R (v. 3.5.1 "Feather Spray") or JASP (v. 0.10.2).

For the DTI analyses, the literature has shown both increases and decreases of fractional anisotropy in psychosis and schizophrenia, although most studies appear to report increases $[35,36]$. Since the analyses were exploratory, we opted to make use of a two-sided $t$-test, without any corrections for multiple comparisons. P-values from these analyses are hence to be considered only as continuous assessments of indirect evidence against the null, and not of confirmatory nature [37]. Since DTI data was only available for a subset of the subjects, matching was not possible and the analysis was unpaired. For information on patient-control matching see Supplementary information.

\section{RESULTS}

Patients were moderately ill, with average PANSS and CGI scores of 65.6 and 4.5 respectively, and the duration of illness was 12.1 months. Additional demographic, radiochemical, and clinical variables for patients and healthy controls are presented in Table 1.

\section{Analysis of D2-R availability in the whole thalamus}

The frequentist paired $t$-test showed a significant difference between patients and healthy controls in $\left[{ }^{11} \mathrm{C}\right] \mathrm{FLB} 457 \mathrm{BP}_{\mathrm{ND}}$ in the whole-thalamus ROI, with patients having lower binding 
Table 1. Descriptive demographic, radiochemical, and clinical data for patients and healthy control subjects.

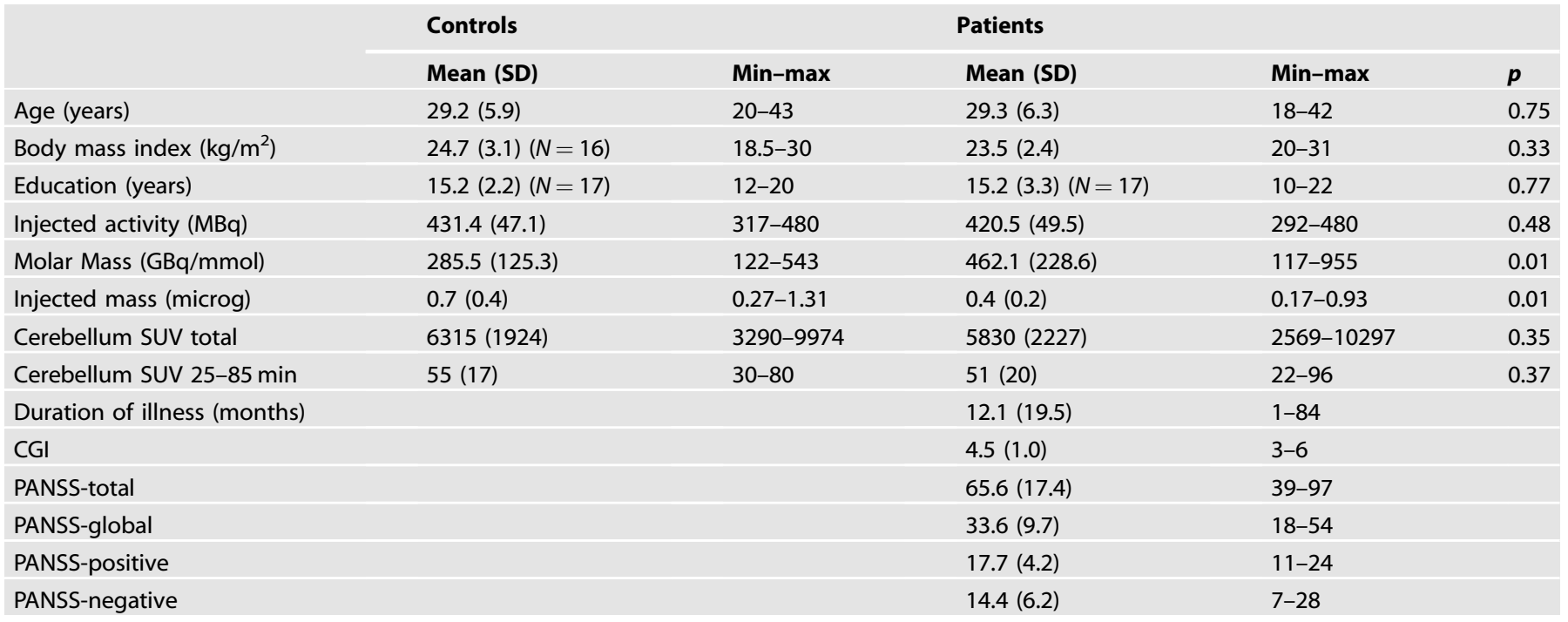

SUV Standardized uptake values, CGI Clinical Global Impressions Scale, PANSS Positive and Negative Syndrome Scale.

compared with healthy controls (Table 2 and Fig. 2). The $\mathrm{BF}_{\mathrm{H} 1: \mathrm{HO}}$ from the Bayesian paired $t$-test indicated that the data was $\sim 5$ times more likely to have originated under the alternative hypothesis compared to the null hypothesis of no difference.

\section{Meta-analyses}

When combining the results from this study with previous literature on D2-R availability differences in the whole thalamus, the meta-analysis showed a significant overall lower binding in patients with psychotic disorders compared with healthy controls (pooled effect size -0.29 , Fig. 3a). This was also the case when including only studies performed using the two high-affinity radioliogands $\left[{ }^{18} \mathrm{~F}\right]$ Fallypride and $\left[{ }^{11} \mathrm{C}\right] \mathrm{FLB} 457$, with a slightly larger pooled effect size of -0.37 (Fig. 3b).

\section{Analysis of D2-R availability in thalamic subregions}

Among the thalamic subregions defined by the Oxford Thalamic Connectivity atlas, the thalamus-PFC ROI showed the largest effect (Table 2), with $\mathrm{BF}_{\mathrm{H} 1: \mathrm{HO}}$ indicating that there was 6 times more support for the alternative hypothesis compared to the null hypothesis (Table 2 and Fig. 2). The difference was also significant with a $p$ value $(0.017)$ below the family-wise error rate corrected alpha threshold. No other subregion in the thalamus showed significant differences, and support for either hypothesis was inconclusive according to the Bayesian paired $t$-tests $\left(\mathrm{BF}_{\mathrm{H} 1: \mathrm{HO}}\right.$ and $\left.\mathrm{BF}_{\mathrm{HO}: \mathrm{H} 1}<3\right)$.

\section{DTI analysis}

The final objective of the study was to explore possible differences in structural thalamocortical connectivity using DTI, with fractional anisotropy of the white matter tracts as the parameter of interest. For the ITR, data were skewed and the non-parametric Mann-Whitney $U$-test was used when comparing patients and healthy subjects. Patients showed lower fractional anisotropy values (mean $=0.375,0.017 \mathrm{SD}$ ) compared with healthy control subjects (mean $=0.389,0.020$ SD, Cohen's $d=-0.692, p=0.036$ ) in the ITR, whereas fractional anisotropy values of the ATR showed very similar values in patients (mean $=0.423,0.0145 \mathrm{SD}$ ) and controls (mean $=$ $0.426,0.023$ SD, Cohen's $d=0.128, p=0.75$ ).

\section{PANSS correlations}

Associations were explored between the PANSS total, PANSS positive and PANSS negative scores and $\mathrm{BP}_{\mathrm{ND}}$ in the whole thalamus and in the Thalamus-PFC subregion, finding no significant correlations (see Supplementary information).

\section{Robustness checks}

After observing a significant difference between patients and controls in injected mass (Table 1) we examined the association between injected mass, specific molar activity, and $\left[{ }^{11} \mathrm{C}\right] \mathrm{FLB} 457$ $\mathrm{BP}_{\mathrm{ND}}$, finding no association (see Supplementary information). No significant difference was observed between the cerebellar SUV of patients and controls (Supplementary Table 1). There was also no difference in thalamic volumes between patients and controls (Supplementary Table 2).

Two-sample (unpaired) $p$-values for the $\mathrm{BP}_{\mathrm{ND}}$ patient-control comparisons in whole thalamus and thalamic subregions showed similar results as in the main analysis (Supplementary Table 3). We also re-ran the main analyses after having excluded four participants with partially incomplete or missing data, finding somewhat higher $p$-values but unchanged effect sizes (Supplementary Table 4).

We performed a set of robustness-checks over the alternative hypotheses in the BF $t$-tests. These also provided evidence in favor of alternative hypothesis of lower D2-R availability in patients (Supplementary Table 5, Supplementary Fig. 3, and Supplementary information).

Funnel plots for the meta-analyses are shown in Supplementary Fig. 4, revealing no apparent publication bias.

Finally, we performed an additional meta-analysis excluding Talvik et al. [38] and Yasuno et al. [10], since there was some overlap in the sample between these studies and Talvik et al. [12] and Suhara et al. [14], respectively. This exclusion did not change the overall finding of lower thalamic D2-R availability in patients compared with healthy controls (Supplementary Fig. 5).

\section{DISCUSSION}

In this PET study examining thalamic D2-R in a sample of only drug-naive psychosis patients compared to healthy controls we observed lower binding in patients. This is the first study investigating thalamic subregions in psychosis patients using high-resolution PET. By employing a connectivity-based atlas we identified lower D2-R levels in a thalamic subregion with projections to the frontal cortex. In a subgroup of patients, we 
observed a tentative result of lower fractional anisotropy values compared with controls in the ITR, which predominantly projects to and from the thalamus and the orbitofrontal, temporal and insular cortex. Taken together, the PET results strengthen the evidence for changes in dopaminergic neurotransmission in the thalamus as a part of the pathophysiology in schizophrenia. Based on the exploratory DTI analyses, we further hypothesize that these may underlie aberrant connectivity between regions shown to be of importance for the pathophysiology of the disorder. Notably, an increase or no change in striatal D2-R has been observed in schizophrenia and psychosis patients [4], suggestive of regionally specific alterations of dopaminergic transmission.

Previous studies investigating $D 2-R$ in thalamus have yielded inconsistent results. Two early studies using $\left[{ }^{11} \mathrm{C}\right] \mathrm{FLB} 457$ in drugnaive samples showed lower D2-R binding in the thalamus $[10,12]$. The sample in Talvik et al. [12] was then extended to 18 patients in a study using the medium affinity radioligand $\left[{ }^{11} \mathrm{C}\right]$ raclopride showing lower $\mathrm{D} 2-\mathrm{R}$ in the right thalamus compared with controls [38]. A more recent study using $\left[{ }^{11} \mathrm{C}\right] \mathrm{FLB} 457$ in a combination of drug naive and drug free patient showed no difference in thalamic binding [13]. In two studies, single photon emission computer tomography (SPECT) and the radioligand $\left[{ }^{123} \mathrm{I}\right]$ epidepride was used in 25 and 6 antipsychotic-naive patients respectively, showing no significant difference in binding between patients and controls $[39,40]$. Two studies that employed PET and the radioligand $\left[{ }^{18} \mathrm{~F}\right]$ Fallypride in a combination of drug-naive and drug-free patients, showed lower $\mathrm{BP}_{\mathrm{ND}}$ values medial thalamic subregions $[9,11]$ whereas one study showed an increase in whole thalamus [16] and yet another one found no difference [15]. Finally, two studies using the agonist and D3-R preferring radioligand $\left[{ }^{11} \mathrm{C}\right] \mathrm{PHNO}$ in 13 drug-free and 12 drug-naive patients respectively showed no significant difference compared with controls [41, 42].

In these studies, sample sizes have generally been small, and several studies have had methodological limitations. SPECT has much lower spatial resolution than PET, a factor which is critical when examining smaller structures in the brain. Of the radioligands used, $\left[{ }^{18} \mathrm{~F}\right]$ Fallypride and $\left[{ }^{11} \mathrm{C}\right] \mathrm{FLB} 457$ are highaffinity tracers, while $\left[{ }^{11} \mathrm{C}\right]$ raclopride shows low specific binding and poor convergent validity in thalamus [43, 44]. Importantly, several studies included patients previously treated with antipsychotics $[9,11,13,15,16,41]$, which has been shown to result in increased D2-R levels in post-mortem and in vivo studies [45, 46]. Thus, medication effects may have masked "true" reductions in baseline D2-R in some previous studies. In the present study we exclusively included antipsychotic-naive patients, and used highresolution PET in combination with a high-affinity radioligand for D2-R. To inform our analysis, we used all previously published data on differences between healthy controls and drug-free patients with psychotic disorders or schizophrenia, confirming support in favor of decreased D2-R availability in patients. Finally, by adding the results from this study to the previous studies in a metaanalysis, we show an overall significantly lower D2-R availability in patients (Fig. 3) (despite possible medication effects in previous studies), which is major step towards resolving the uncertainty in the previous literature.

It should be noted however that the overlap between the patient and healthy control group is large in both the current study and in the meta-analyses, with the $95 \% \mathrm{Cls}$ being both compatible with a medium sized, as well as a negligible groupdifference. This suggests that a subgroup of patients may differ in thalamic D2-R receptor levels compared with healthy participants, but that there will also be patients in the population showing little to no aberration. Further studies in larger samples are needed to identify such subgroups.

The thalamic complex includes a large number of cytoarchitectonically distinct nuclei, each being components of circuits with distinct physiological and functional implications [22,47]. Whereas 

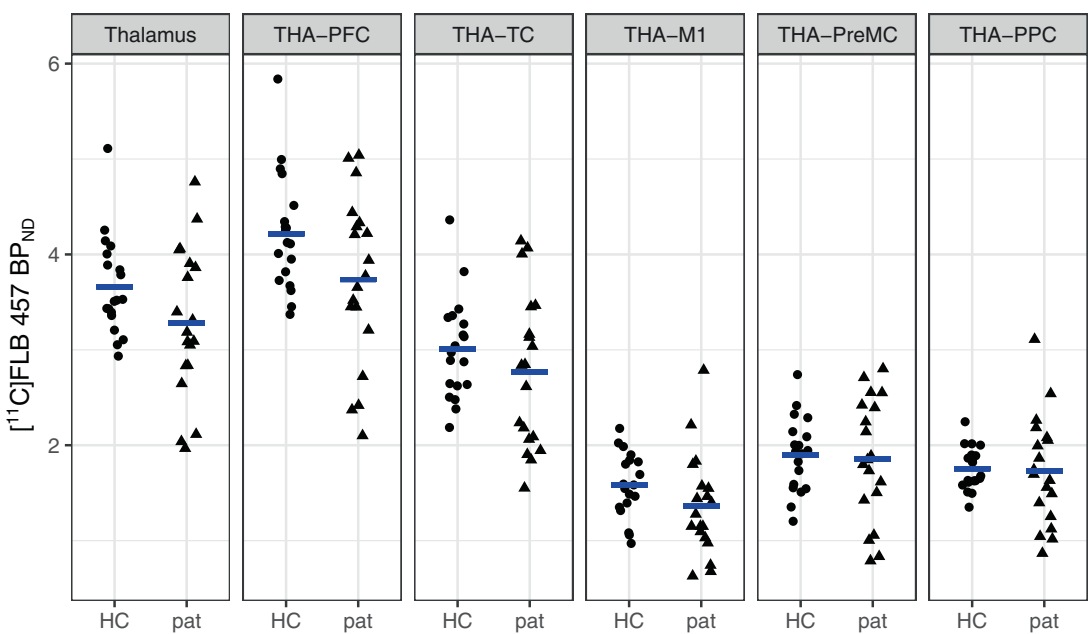

Fig. 2 Patient-control differences in $\left[{ }^{11} \mathrm{C}\right] \mathrm{FLB} 457 \mathrm{BP}_{\mathrm{ND}}$ in the whole thalamus and subthalamic regions. There was a significant difference between groups in the whole thalamus as well as the subregion corresponding to prefrontal cortex connectivity.

\section{A. All studies}

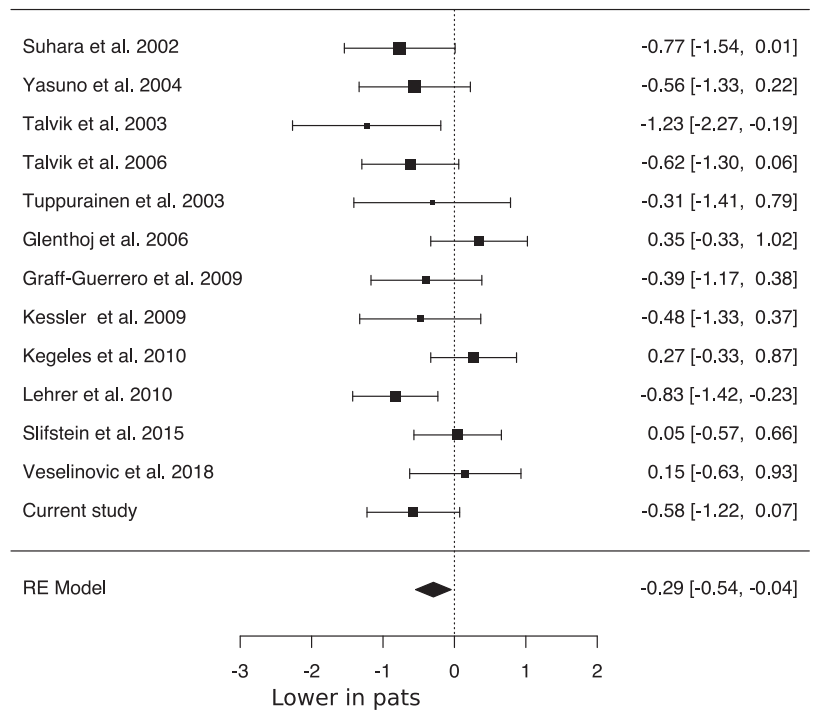

B. $\left[{ }^{18} \mathrm{~F}\right]$ Fallypride and $\left[{ }^{11} \mathrm{C}\right] \mathrm{FLB} 457$

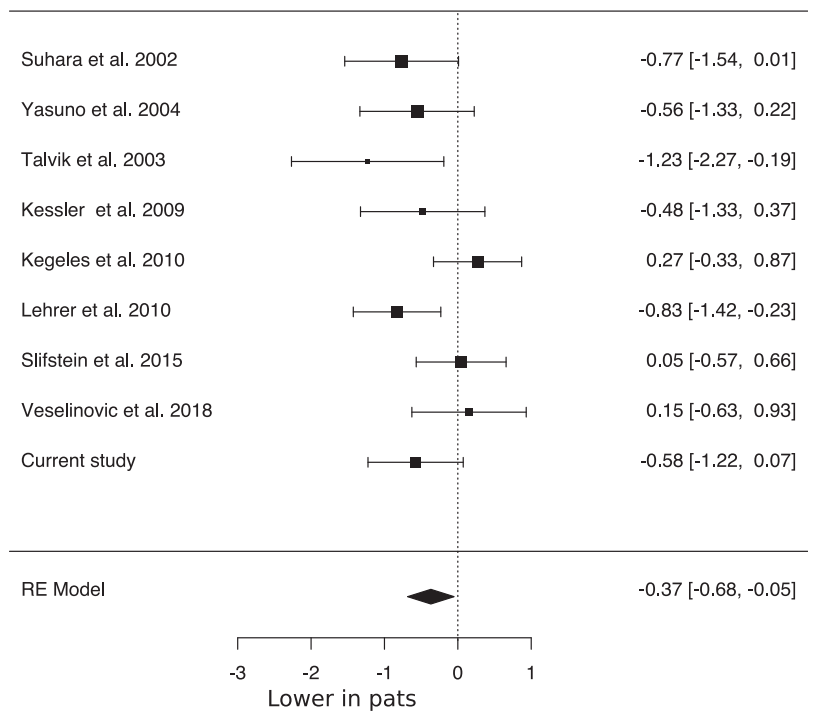

Fig. 3 Meta-analyses of thalamic D2-R availability differences between patients and controls. A Patients show significantly overall lower D2-R levels in whole thalamus compared with healthy control subjects. B Meta-analysis only including studies which used the two highaffinity radioligands $\left[{ }^{11} \mathrm{C}\right] \mathrm{FLB} 457$ and $\left[{ }^{18} \mathrm{~F}\right]$ Fallypride. Additional meta-analyses excluding studies with partially overlapping samples, and funnel plots can be found in the supplementary information.

previous studies have explored arbitrarily defined thalamic subregions, we used an atlas of thalamic subregions based on DTI connectivity data [22], which in combination with highresolution PET provides an increase in functional-anatomical specificity. Our results are consistent with aberrant dopaminergic functioning in the subregion relaying to the prefrontal cortex. The frontal cortex is believed to play an important role in the pathology behind psychotic disorder, and has been centrally implicated in both positive, negative and cognitive symptoms of schizophrenia [48-50].

From a functional neuroanatomical perspective, the frontal cortex is a large and heterogenous area. Using DTI we attempted to be more specific, assuming that altered receptor levels are related to alterations in structure that is reflected in the white matter structure and DTI metrics. Our combined PET (pointing at prefrontal cortex) and DTI results suggest changes in DA neurotransmission in thalamocortical circuits involving more specific parts of the prefrontal cortex, namely the orbitofrontal cortex. It also allows us to hypothesize an involvement of the frontal insular cortex, which is included in the thalamus-PFC ROI used [22]. In contrast, we did not observe any differences in the ATR, which has been implicated in other DTI studies on antipsychotic-naive first-episode psychosis patients [51]. Orbitofrontal cortex and insula have both shown structural changes in antipsychotic-naive schizophrenia subjects as measured with voxel-based morphometry $[52,53]$ and are central to in reward processing and self-awareness, two functions that are thought to be perturbed in schizophrenia [50,54].

A possible factor underlying the observed changes in dopamine receptor levels may be loss of synaptic connections. Post-mortem studies have shown widespread loss of synaptic structures, as well as reduction of synaptic markers in schizophrenia $[55,56]$. Recently a PET study employing the radioligand $\left[{ }^{11} \mathrm{C}\right] \mathrm{UCB}-\mathrm{J}$, which binds to the synaptic vesicle glycoprotein $2 \mathrm{~A}$ showed widespread 
lower binding in patients compared with controls, indicating lower synaptic density in cortical regions as well as the thalamus [57]. Further, mechanistic data based on patient-derived cellular models indicate increased microglial synapse elimination in schizophrenia and link the schizophrenia risk locus containing the complement component 4 isotype genes to excessive synapse elimination [58]. This motivates further investigations into the relationship between synaptic loss and connectivity in thalamocortical projections, and dopaminergic function in schizophrenia.

A potential limitation of the present analysis is that a series of studies have shown that a small amount of specific $\left[{ }^{11} \mathrm{C}\right] \mathrm{FLB} 457$ binding is present in the cerebellum for most subjects [59-61], such that a more ideal quantification of binding would be to measure radioactivity in arterial blood to use as an arterial input function. Although non-significant differences between the cerebellar SUV of patients and controls were observed, cerebellar binding cannot be fully excluded as a potential confounder. Another possible confounder is that of partial volume effects, meaning that smaller volumes in patients could lead to increased loss of signal, resulting in false low $\mathrm{BP}_{\mathrm{ND}}$ values. However, volumes for whole thalamus as well as thalamic subregions were numerically higher in this specific patient sample, making this explanation unlikely. Finally, 5 patients had received low doses of bensodiazepines at the time of PET which may have influenced the results [62]. Apart from the limitation constituted by the modest sample size, there are technical constraints in the DTI analysis such as the low and anisotropic resolution. In addition, tractography of the ITR suffers from its orbitofrontal course which is a distortion-prone region in MRI. Our anatomical description is however in line with that of other researchers using DTI $[22,28]$ and the animal literature [27].

In conclusion, our findings support the hypothesis of a dysregulation of thalamic dopaminergic neurotransmission in schizophrenia. Based on the results, we hypothesize that such dopaminergic aberrations could in turn could underlie a disturbance of specific thalamocortical circuits. This should be investigated in future studies, including a larger sample of drugnaive patients examined with DTI.

\section{DATA AVAILABILITY}

Due to institutional restrictions, the data of this project cannot be shared on a public repository. Instead, the data can be made available upon request on a case by case basis as allowed by the legislation and ethical permits. Requests for access can be made to the Karolinska Institutet's Research Data Office at rdo@ki.se. The R analysis code and preregistration protocol can be found on https://osf.io/nhr3w/.

\section{REFERENCES}

1. Nord M, Farde L. Antipsychotic occupancy of dopamine receptors in schizophrenia. CNS Neurosci Ther. 2011;17:97-103.

2. Farde L, Nordström AL, Wiesel FA, Pauli S, Halldin C, Sedvall G. Positron emission tomographic analysis of central D1 and D2 dopamine receptor occupancy in patients treated with classical neuroleptics and clozapine: relation to extrapyramidal side effects. Arch Gen Psychiatry. 1992;49:538-44.

3. Ripke S, Neale BM, Corvin A, Walters JTR, Farh K-H, Holmans PA, et al. Biological insights from 108 schizophreniaassociated genetic loci. Nature. 2014;511:421-7.

4. Howes OD, Kambeitz J, Kim E, Stahl D, Slifstein M, Abi-Dargham A, et al. The nature of dopamine dysfunction in schizophrenia and what this means for treatment. Arch Gen Psychiatry. 2012;69:776-86.

5. Hwang K, Bertolero MA, Liu WB, D'Esposito M. The human thalamus is an integrative hub for functional brain networks. J Neurosci. 2017;37:5594-607.

6. Wagner G, De la Cruz F, Schachtzabel C, Güllmar D, Schultz CC, Schlösser RG, et al. Structural and functional dysconnectivity of thefronto-thalamic system in schizophrenia: ADCM-DTI study. Cortex 2015;66:35-45.

7. Halldin C, Farde L, Hogberg T, Mohell N, Hall H, Suhara T, et al. Carbon-11-FLB 457: A radioligand for extrastriatal D2 dopamine receptors. J Nucl Med. 1995;36:1275-81.

8. Mukherjee J, Yang ZY, Das MK, Brown T. Fluorinated benzamide neuroleptics-III. Development of (S)-N-[(1-allyl-2-pyrrolidinyl)methyl]-5-(3-[18F]fluoropropyl)- 2,3-dimethoxybenzamide as an improved dopamine D-2 receptor tracer. Nucl Med Biol. 1995;22:283-96.

9. Lehrer DS, Christian BT, Kirbas C, Chiang M, Sidhu S, Short H, et al. 18F-Fallypride binding potential in patients with schizophrenia compared to healthy controls. Schizophr Res. 2010;122:43-52.

10. Yasuno $F$, Suhara $T$, Okubo $Y$, Sudo $Y$, Inoue $M$, Ichimiya $T$, et al. Low dopamine d (2) receptor binding in subregions of the thalamus in schizophrenia. Am J Psychiatry. 2004;161:1016-22.

11. Kessler RM, Woodward ND, Riccardi P, Li R, Ansari MS, Anderson S, et al. Dopamine D2 Receptor Levels in Striatum, Thalamus, Substantia Nigra, Limbic Regions, and Cortex in Schizophrenic Subjects. Biol Psychiatry. 2009;65:1024-31.

12. Talvik M, Nordström AL, Olsson H, Halldin C, Farde L. Decreased thalamic D2/D3 receptor binding in drug-naive patients with schizophrenia: A PET study with [11C]FLB 457. Int J Neuropsychopharmacol. 2003;6:361-70.

13. Slifstein M, van de Giessen E, Van Snellenberg J, Thompson JL, Narendran R, Gil R, et al. Deficits in prefrontal cortical and extrastriatal dopamine release in schizophrenia: a positron emission tomographic functional magnetic resonance imaging study. JAMA Psychiatry. 2015;72:316-24.

14. Suhara T, Okubo $Y$, Yasuno F, Sudo $Y$, Inoue $M$, Ichimiya $T$, et al. Decreased dopamine D2 receptor binding in the anterior cingulate cortex in schizophrenia. Arch Gen Psychiatry. 2002;59:25-30.

15. Veselinović T, Vernaleken I, Janouschek H, Cumming P, Paulzen M, Mottaghy FM, et al. The role of striatal dopamine D $2 / 3$ receptors in cognitive performance in drug-free patients with schizophrenia. Psychopharmacology. 2018;235:2221-32.

16. Kegeles LS, Slifstein M, Xu X, Urban N, Thompson JL, Moadel T, et al. Striatal and Extrastriatal Dopamine D2/D3 Receptors in Schizophrenia Evaluated With [18F] fallypride Positron Emission Tomography. Biol Psychiatry. 2010;68:634-41.

17. Kambeitz J, Abi-Dargham A, Kapur S, Howes OD. Alterations in cortical and extrastriatal subcortical dopamine function in schizophrenia: systematic review and meta-analysis of imaging studies. Br J Psychiatry. 2014;204:420-9.

18. Byne W, Hazlett EA, Buchsbaum MS, Kemether E. The thalamus and schizophrenia: current status of research. Acta Neuropathol. 2009;117:347-68.

19. Pakkenberg B. Pronounced Reduction of Total Neuron Number in Mediodorsal Thalamic Nucleus and Nucleus Accumbens in Schizophrenics. Arch Gen Psychiatry. 1990;47:1023-8.

20. Dorph-Petersen KA, Lewis DA. Postmortem structural studies of the thalamus in schizophrenia. Schizophr Res. 2017;180:28-35.

21. Jenkinson M, Beckmann CF, Behrens TEJ, Woolrich MW, Smith SM. Review FSL. Neuroimage 2012;62:782-90.

22. Behrens TEJ, Johansen-Berg H, Woolrich MW, Smith SM, Wheeler-Kingshott CAM, Boulby PA, et al. Non-invasive mapping of connections between human thalamus and cortex using diffusion imaging. Nat Neurosci. 2003;6:750-7.

23. Matheson GJ, Stenkrona $P$, Cselényi $Z$, Plavén-Sigray $P$, Halldin $C$, Farde $L$, et al Reliability of volumetric and surface-based normalisation and smoothing techniques for PET analysis of the cortex: A test-retest analysis using [11C]SCH-23390. Neuroimage. 2017;155:344-53.

24. Andersson J, Truong P, Halldin C. In-target produced [11C]methane: Increased specific radioactivity. Appl Radiat Isot. 2009;67:106-10.

25. Logan J, Fowler JS, Volkow ND, Wolf AP, Dewey SL, Schlyer DJ, et al. Graphical analysis of reversible radioligand binding from time-activity measurements applied to [N-11C-methyl]-(-)-cocaine PET studies in human subjects. J Cereb Blood Flow Metab. 1990;10:740-7.

26. Tjerkaski J, Cervenka S, Farde L, Matheson GJ. Kinfitr-an open source tool for reproducible $\mathrm{PET}$ modelling: validation and evaluation of test-retest reliability. EJNMMI Res. 2020;10:77.

27. Schmahmann JD, Pandaya DN. Fiber pathways of the brain. New York: Oxford University Press; 2006.

28. Wang F, Sun T, Li XG, Liu NJ. Diffusion tensor tractography of the temporal stem on the inferior limiting sulcus: Laboratory investigation. J Neurosurg. 2008;108:775-81.

29. Niida R, Yamagata B, Niida A, Uechi A, Matsuda H, Mimura M. Aberrant anterior thalamic radiation structure in bipolar disorder: A diffusion tensor tractography study. Front Psychiatry. 2018;9:1-7.

30. Leemans $A$, Jones DK. The $B$-matrix must be rotated when correcting for subject motion in DTI data. Magn Reson Med. 2009;61:1336-49.

31. Klein S, Staring M, Murphy K, Viergever M, Pluim J. elastix: a toolbox for intensitybased medical image registration. IEEE Trans Med Imaging. 2010;29:196-205.

32. Kass RE, Raftery AE. Bayes factors. J Am Stat Assoc. 1995;90:773-95.

33. Lee $M$, Wagenmakers E-J. Bayesian cognitive modeling: A practical course. Cambridge: Cambridge University Press; 2014.

34. Nichols TE, Holmes AP. Nonparametric permutation tests for functional neuroimaging: a primer with examples. Hum Brain Mapp. 2002;15:1-25.

35. Kelly S, Jahanshad N, Zalesky A, Kochunov P, Agartz I, Alloza C, et al. Widespread white matter microstructural differences in schizophrenia across 4322 individuals: 
Results from the ENIGMA Schizophrenia DTI Working Group. Mol Psychiatry. 2018;23:1261-9.

36. Barth C, Lonning V, Gurholt TP, Andreassen OA, Myhre AM, Agartz I. Exploring white matter microstructure and the impact of antipsychotics in adolescentonset psychosis. PLoS ONE. 2020;15:e0233684.

37. Perezgonzalez JD. Fisher, Neyman-Pearson or NHST? A tutorial for teaching data testing. Front Psychol. 2015;6:1-11.

38. Talvik M, Nordström A-L, Okubo Y, Olsson H, Borg J, Halldin C, et al. Dopamine D2 receptor binding in drug-naïve patients with schizophrenia examined with raclopride-C11 and positron emission tomography. Psychiatry Res. 2006;148:165-73.

39. Glenthoj BY, Mackeprang T, Svarer C, Rasmussen H, Pinborg LH, Friberg L, et al. Frontal dopamine $D(2 / 3)$ receptor binding in drug-naive first-episode schizophrenic patients correlates with positive psychotic symptoms and gender. Biol Psychiatry. 2006;60:621-9.

40. Tuppurainen H, Kuikka JT, Laakso MP, Viinamaki H, Husso M, Tiihonen J. Midbrain dopamine D2/3 receptor binding in schizophrenia. Eur Arch Psychiatry Clin Neurosci. 2006;256:382-7.

41. Graff-Guerrero A, Mizrahi R, Agid O, Marcon H, Barsoum P, Rusjan P, et al. The Dopamine D2 Receptors in High-Affinity State and D3 Receptors in Schizophrenia: A Clinical [11C]-(+)-PHNO PET Study. Neuropsychopharmacology. 2009;34:1078-86.

42. Suridjan I, Rusjan P, Addington J, Wilson A, Houle S, Mizrahi R. Dopamine D2 and D3 binding in people at clinical high risk for schizophrenia, antipsychotic-naive patients and healthy controls while performing a cognitive task. J Psychiatry Neurosci. 2013;38:98-106.

43. Svensson JE, Schain $M$, Plavén-Sigray $P$, Cervenka $S$, Tiger $M$, Nord $M$, et al. Validity and reliability of extrastriatal [11C]raclopride binding quantification in the living human brain. Neuroimage. 2019;202:116143.

44. Freiburghaus T, Svensson JE, Matheson GJ, Plavén-Sigray P, Lundberg J, Farde L, et al. Low convergent validity of $[11 C]$ raclopride binding in extrastriatal brain regions: A PET study of within-subject correlations with [11C]FLB 457. Neuroimage. 2021;226:1-21.

45. Silvestri S, Seeman MV, Negrete JC, Houle S, Shammi CM, Remington GJ, et al. Increased dopamine D2 receptor binding after long-term treatment with antipsychotics in humans: A clinical PET study. Psychopharmacology. 2000;152:174-80.

46. Seeman P, Bzowej NH, Guan HC, Bergeron C, Reynolds GP, Bird ED, et al. Human brain D1 and D2 dopamine receptors in schizophrenia, Alzheimer's, Parkinson's, and Huntington's diseases. Neuropsychopharmacology. 1987;1:5-15.

47. Morel A, Magnin M, Jeanmonod D. Multiarchitectonic and stereotactic atlas of the human thalamus. J Comp Neurol. 1997;387:588-630.

48. Selemon LD, Goldman-Rakic PS. The reduced neuropil hypothesis: a circuit based model of schizophrenia. Biol Psychiatry. 1999;45:17-25.

49. Cannon TD, Thompson PM, Van Erp TGM, Toga AW, Poutanen VP, Huttunen M, et al. Cortex mapping reveals regionally specific patterns of genetic and diseasespecific gray-matter deficits in twins discordant for schizophrenia. Proc Natl Acad Sci USA. 2002;99:3228-33.

50. Cannon TD. How schizophrenia develops: cognitive and brain mechanisms underlying onset of psychosis. Trends Cogn Sci. 2015;19:744-56.

51. Ebdrup BH, Raghava JM, Nielsen M, Rostrup E, Glenthøj B. Frontal fasciculi and psychotic symptoms in antipsychotic-naive patients with schizophrenia before and after 6 weeks of selective dopamine D2/3 receptor blockade. J Psychiatry Neurosci. 2016;41:133-41.

52. Ellison-Wright I, Glahn DC, Laird AR, Thelen SM, Bullmore E. The anatomy of firstepisode and chronic schizophrenia: An anatomical likelihood estimation metaanalysis. Am J Psychiatry. 2008;165:1015-23.

53. Haijma SV, Van Haren N, Cahn W, Koolschijn PCMP, Hulshoff Pol HE, Kahn RS. Brain volumes in schizophrenia: a meta-analysis in over 18000 subjects. Schizophr Bull. 2013;39:1129-38.

54. Roiser JP, Stephan KE, den Ouden HEM, Friston KJ, Joyce EM. Adaptive and aberrant reward prediction signals in the human brain. Neuroimage 2010;50:657-64.

55. Glausier JR, Lewis DA. Dendritic spine pathology in schizophrenia. Neuroscience 2013;251:90-107.

56. Osimo EF, Beck K, Reis Marques T, Howes OD. Synaptic loss in schizophrenia: a meta-analysis and systematic review of synaptic protein and mRNA measures. Mol Psychiatry. 2019;24:549-61.

57. Onwordi EC, Halff EF, Whitehurst T, Mansur A, Cotel MC, Wells L, et al. Synaptic density marker SV2A is reduced in schizophrenia patients and unaffected by antipsychotics in rats. Nat Commun. 2020;11:246.

58. Sellgren CM, Gracias J, Watmuff B, Biag JD, Thanos JM, Whittredge PB, et al. Increased synapse elimination by microglia in schizophrenia patient-derived models of synaptic pruning. Nat Neurosci. 2019;22:374-85.

59. Narendran R, Mason NS, Chen C-M, Himes M, Keating P, May MA, et al. Evaluation of dopamine D2/3 specific binding in the cerebellum for the positron emission tomography radiotracer $\left[{ }^{11} \mathrm{C}\right] \mathrm{FLB}$ 457: implications for measuring cortical dopamine release. Synapse. 2011;65:991-7.

60. Asselin M-C, Montgomery AJ, Grasby PM, Hume SP. Quantification of PET studies with the very high-affinity dopamine D2/D3 receptor ligand [11C]FLB 457: reevaluation of the validity of using a cerebellar reference region. J Cereb Blood Flow Metab. 2007;27:378-92.

61. Olsson H, Halldin C, Farde L. Differentiation of extrastriatal dopamine D2 receptor density and affinity in the human brain using PET. Neuroimage. 2004;22:794-803.

62. Vilkman H, Kajander J, Aalto S, Vahlberg T, Någren K, Allonen T, et al. The effects of lorazepam on extrastriatal dopamine D2/3-receptors-A double-blind randomized placebo-controlled PET study. Psychiatry Res - Neuroimaging. 2009;174:130-7.

\section{ACKNOWLEDGEMENTS}

We thank research nurses Joachim Eckerström, Marie Adolfsson, Minna Juntura, Martin Szabo, Henrik Gregemark; the staff at Psykiatri Nordväst, Norra Stockholms Psykiatri, PRIMA Vuxenpsykiatri; and the staff of the PET group at Karolinska Institutet for their invaluable assistance during this study. AS wishes to thank Markus Nilsson and Filip Szczepankiewicz at Lund University for ExploreDTI support. The study was supported by the Swedish Research Council (Grant No. 523-2014-3467 (SC), 2017-00875 (SE), 09114 (LF), K2015-62X-15077-12-3, 2017-00949 (IA)), Karolinska Institutet and Stockholm County Council ((SC, LF, SE, CMS)) and Torsten Söderberg Stiftelse (SE). PPS was supported by the Swedish Society for Medical Research and the Lundbeck Foundation. AF was supported by Swedish Society for Medical Research and The Benthe Rexhed Gersteds foundation.

\section{AUTHOR CONTRIBUTIONS}

$\mathrm{LF}, \mathrm{SC}, \mathrm{IA}, \mathrm{HFB}$, and $\mathrm{CH}$ conceived of the study. PPS, PV, LF, and SC designed the study. PV, KC, SC, ML, IA, collected the data. CH was responsible for radiochemistry. PPS and PV carried out the image analysis of PET data. AFS carried out the image analysis of DTI data. PPS and SC conducted the literature search. PPS and GJM carried out the statistical analyses of the PET data. AFS carried out the statistical analyses of the DTI data. PPS, AFS, PV, ML, and SC drafted the article. All authors interpreted the results, critically revised the article and approved of the final version for publication.

\section{COMPETING INTERESTS}

SC has served as a one-off speaker for Otsuka Pharmaceuticals. LF was at the time of data collection partially employed at the AstraZeneca PET imaging Centre at Karolinska Institutet. CMS is a scientific advisor to Outermost Therapeutics Inc. None of the authors declare conflict of interest in relation to the present work.

\section{ADDITIONAL INFORMATION}

Supplementary information The online version contains supplementary material available at https://doi.org/10.1038/s41380-021-01349-x.

Correspondence and requests for materials should be addressed to Simon Cervenka.

Reprints and permission information is available at http://www.nature.com/ reprints

Publisher's note Springer Nature remains neutral with regard to jurisdictional claims in published maps and institutional affiliations. Attribution 4.0 International License, which permits use, sharing, adaptation, distribution and reproduction in any medium or format, as long as you give appropriate credit to the original author(s) and the source, provide a link to the Creative Commons license, and indicate if changes were made. The images or other third party material in this article are included in the article's Creative Commons license, unless indicated otherwise in a credit line to the material. If material is not included in the article's Creative Commons license and your intended use is not permitted by statutory regulation or exceeds the permitted use, you will need to obtain permission directly from the copyright holder. To view a copy of this license, visit http://creativecommons. org/licenses/by/4.0/.

(c) The Author(s) 2021 